\title{
La aplicación de tecnologías regtech en el cumplimiento del deber de información de los emisores de valores en Colombia
}

DOI: http://dx.doi.org/10.15425/2017.514

\section{Resumen}

El deber de información de los emisores de valores, si bien propende por la transparencia y la eficiencia de los mercados, implica costos económicos, jurídicos y comerciales. Este artículo demuestra que, desde el marco de la intervención estatal en el mercado de valores en Colombia, el uso de las nuevas tecnologías de la información en el cumplimiento de los deberes regulatorios y en la supervisión de este, conocidas en este contexto como tecnologías regtech, permite que los emisores de valores cumplan el deber de información. Con ese propósito, el artículo planteará expedir una nueva regulación que, probada mediante las tecnologías propuestas, combine la simplificación de la información que se reporta con las nuevas tecnologías, y la correcta mitigación de los riesgos que conlleva su aplicación.

\section{Palabras clave}

Deber de información de los emisores de valores, cumplimiento regulatorio, regtech.

\footnotetext{
Abogado de la Pontificia Universidad Javeriana, especialista en legislación financiera y magíster en Derecho Privado de la Universidad de los Andes, con experiencia en derecho bancario, del mercado de capitales y corporativo. Actualmente es abogado del Área de Mercado de Capitales de Ustáriz \& Abogados Estudio Jurídico. davidgortizr@gmail.com

El autor quiere extender sus más sinceros agradecimientos a todas las personas que de una u otra forma hicieron posible que este artículo, que pasó de ser un trabajo de grado a un proyecto de todos los días, viera la luz. Un agradecimiento muy especial a Germán Ortiz Pérez y María Lilian Rey Moreno, artífices y fervientes creyentes de este proyecto llamado David Guillermo.
} 
The application of regtech technologies in the compliance of issuers' disclosure duty in Colombia

\begin{abstract}
Disclosure duty, even if it intends to promote transparency and efficiency in capital markets, implies economic, legal, and commercial costs for issuers. This paper demonstrates that, within the framework of state intervention in Colombian capital markets, the application of new technological instruments in compliance and in real time monitoring, known as regtech, guarantees the fulfilling of issuers disclosure duty. To this end, there will be proposed a new regulation by the Colombian financial authority which, proved through these technologies, combines a simplification of the information to be reported, a combination of technological innovation tools and a correct mitigation of the risks involved.
\end{abstract}

\title{
Keywords
}

Issuers' disclosure duty, regulatory compliance, regtech. 


\section{Introducción}

De acuerdo con la normatividad financiera nacional e internacional, el propósito de la regulación financiera es la protección de los inversionistas, garantizar que los mercados sean justos, eficientes y transparentes y reducir el riesgo sistémico ${ }^{1}$. En ese sentido, uno de los elementos más importantes de la regulación financiera es el deber de información de los emisores de valores.

Este deber se ha establecido como una herramienta que busca garantizar que los precios de los valores se formen de acuerdo con la realidad del mercado y sean un reflejo de la situación de las empresas, que demuestre que los emisores de valores cuentan con una estructura de gobierno corporativo sólida y con políticas que propenden por el trato equitativo de sus accionistas y por la transparencia en el mercado de valores ${ }^{2}$. No obstante, el cumplimiento del deber de información implica para los emisores de valores costos económicos, jurídicos y comerciales. Por este motivo, la utilización de tecnologías que se basan en la ciencia de datos se presenta como una alternativa para potenciar el aprovechamiento, la sistematicidad y la interconectividad de la información transmitida ${ }^{3}$.

El presente artículo pretende demostrar que, desde el marco actual de la intervención estatal en el mercado de valores en Colombia, el regtech, es decir, la capacidad de los reguladores de realizar un monitoreo continuo en tiempo real, por medio de nuevas herramientas tecnológicas ${ }^{4}$, permite que los emisores de valores cumplan, de una manera más eficiente, el deber de información definido en la regulación colombiana e internacional.

En ese sentido, el objetivo general del presente artículo será demostrar que la aplicación del regtech para el cumplimiento del deber de información de los emisores de valores involucra un cambio en la forma en la que se concibe la información transmitida, que estará orientado a evitar que la transmisión de una misma información deba enviarse más de una vez. Así mismo, se argumentará que los reguladores también pueden beneficiarse de las soluciones regtech, concepto

1 International Organization of Securities Commissions (Iosco). Objectives and principles of securities regulation. international organization of securities commissions, 2010, 5.

Armour, Awrey, Davies, Enriques, Gordon, Mayer, Payne. Principles of financial regulation, 2016, 167.

Micheler, Eva y Anna Whaley. "Regulatory technology: Replacing law with computer code”, 2018, 11-12. Arner, Douglas W., Jànos Barberis y Ross P. Buckley. "FinTech, regtech and the reconceptualization of financial regulation", 382. 
conocido como "regtech para reguladores" o SupTech5, el cual permitirá simplificar la recopilación de información y optimizar la gestión de procesos internos ${ }^{6}$, por ejemplo, mediante la utilización de estas tecnologías para desarrollar ambientes de prueba regulatorios, o sandbox ${ }^{7}$, implementados en colaboración con la industria ${ }^{8}$. Para cumplir dicho objetivo, se propondrán tres objetivos específicos.

En primer lugar, se sugerirá, desde los objetivos y criterios de la intervención establecidos en la ley colombiana, una regulación basada en datos seguros y fácilmente transmisibles que permita potenciar el cumplimiento del deber de información de los emisores. Dicho objetivo tendrá en cuenta, primero, la información que deben reportar los emisores de acuerdo con la normatividad vigente. Además, se analizarán las razones por las que las empresas desisten de ser emisoras de valores y las alternativas que se han desarrollado con el fin de cumplir el deber de información de una forma más eficiente y organizada.

Por otro lado, se pondrá de presente la oportunidad que representa para el mercado de valores utilizar las tecnologías regtech en pro de la eficiencia y la transparencia del mercado. Con ese propósito, se expondrán algunas de las tecnologías sobre las que se basa el regtech y su aplicación para el cumplimiento del deber de información. Se introducirá además una funcionalidad del regtech que permitirá desarrollar espacios controlados de prueba de las tecnologías que serán aplicadas

La tecnología de supervisión (suptech o supervision technology) consiste en el uso de tecnología innovadora por parte de las agencias de apoyo a la supervisión, el cual ayuda a las agencias de supervisión a digitalizar informes y procesos regulatorios. De acuerdo con expertos, el suptech podría cambiar las reglas del juego en la generación de informes eficientes y en el monitoreo proactivo del riesgo y cumplimiento de las instituciones financieras, convirtiendo la supervisión de riesgos y el cumplimiento de una perspectiva retrospectiva a un proceso predictivo. Véase Broeders, Dirk y Jermy Prenio. "Innovative technology in financial supervision (suptech) - the experience of early users", 2018, 3.. Herrera, Diego y Sonia Vadillo. "Regulatory sandboxes in Latin America and the Caribbean for the FinTech ecosystem and the financial system", 2018, 21.

Los sandbox (o arenera) son entornos virtuales que se utilizan para probar y examinar los impactos de las innovaciones en procesos o tecnologías de forma aislada. La Autoridad de Conducta Financiera del Reino Unido (FCA) ha liderado el progreso en esta área, con su unidad Project Innovate, anunciando que los sandbox son un "espacio seguro en el que las empresas pueden probar productos, servicios y negocios innovadores modelos y mecanismos de entrega sin incurrir inmediatamente en todas las consecuencias normativas normales de participar en la actividad en cuestión", Arner, Barberis, Buckley, Fintech and Regtech in a nutshell, and the future in a sandbox, 2017, 15. El Ministerio de Hacienda y Crédito Público expidió el Decreto 1234 del 2020 por medio del cual se reglamentaron los requisitos, características y demás elementos relacionados con el espacio controlado de prueba (ECP), sobre el cual la SFC aún está pendiente, para la fecha de publicación de este artículo, de impartir las instrucciones correspondientes. (Herrera, Vadillo, Regulatory Sandboxes in Latin, 21), se utilizará indistintamente la denominación Regtech hacer referencia a la utilización de nuevas tecnologías, tanto por la industria para hacer más eficiente el cumplimiento regulatorio (regtech), como por los reguladores para fortalecer su seguimiento y monitoreo (suptech o regtech para reguladores). 
para el monitoreo del cumplimiento del deber de información. Por último, se realizará un análisis crítico de los riesgos que conlleva dicha aplicación.

En tercer lugar, se propondrá un marco para la utilización en Colombia de las tecnologías regtech, que tendrá como propósito la sistematización y la estandarización de los procesos de cumplimiento. Así, se presentará una propuesta de nueva regulación a expedir por parte de la Superintendencia Financiera de Colombia que aproveche los mejores elementos del regtech para la transmisión de la información, con el propósito de que esta sea entregada de acuerdo con los requerimientos legales, con las políticas de seguridad de la información y ciberseguridad, y evitando duplicidades en la transmisión. Por último, se sugerirán mecanismos que cubran los riesgos de la aplicación del regtech, puesto que la correcta mitigación de estos será fundamental para garantizar la eficiencia en el cumplimiento del deber de información y en su supervisión.

Dicha propuesta expondrá los pasos a seguir tanto por el emisor que debe cumplir su deber de información, como aquellos que debe ejecutar la autoridad de supervisión, dentro del nuevo escenario de cumplimiento presentado. Estos pasos se enfocarán en el cumplimiento de la información que se va a transmitir con respecto a los deberes establecidos en las normas correspondientes, y en el ajuste de dicha información a los estándares técnicos e informáticos para cada una de las tecnologías dispuestas, de acuerdo con los postulados de la ciencia de datos.

Así, el artículo concluirá que la utilización de estas herramientas generará eficiencias en el cumplimiento del deber de información, reduciendo los costos de cumplimiento y eventualmente motivando a nuevas empresas a convertirse en emisores de valores. Para esto será necesario una coordinación entre las autoridades y la industria, un cambio en la forma en la que se analiza el cumplimiento del deber de información, un análisis costo-beneficio que además involucre un estudio de la factibilidad de la aplicación de estas tecnologías por parte de las distintas empresas, y el reconocimiento de las limitaciones que tiene la tecnología para garantizar dicho cumplimiento.

\section{Regulación del deber de información de los emisores de valores}

Los emisores de valores son empresas que recaudan capital en el mercado de valores. La doctrina ha demostrado que existen diferentes ventajas en convertirse en 
emisor de valores ${ }^{9}$. En primer lugar, la obtención de fondos mediante una oferta pública representa un eficiente mecanismo de financiación y fuente de crecimiento de las empresas que permite la realización de sus planes de expansión, así como el incremento de su capital de trabajo y la adquisición de otros negocios. Además, el acceso al mercado aumenta el poder de negociación y el prestigio de la empresa, que puede pasar de ser concebida como una compañía pequeña a una grande y exitosa.

En vista de este panorama, esta parte se referirá a la razón de ser de la regulación de los emisores, a la regulación del deber de información y al modo en que se cumple dicho deber en Colombia.

\section{A) Razón de ser de la regulación de los emisores de valores}

Como parte de la regulación financiera, la regulación de los emisores de valores tiene como propósitos la transparencia y la eficiencia de los mercados, dado que un mercado eficiente debe asegurar que en cualquier momento los inversionistas, incluso aquellos menos informados, adquieran valores en el mercado a un precio justo y no distorsionado ${ }^{10}$. Esto favorece la liquidez de los mercados de capitales y brinda eficiencia en las inversiones y en el manejo de la información.

Es así como en la regulación colombiana, la Ley 964 del 2005 establece que el Gobierno nacional ejercerá la intervención en las actividades de manejo, aprovechamiento e inversión de recursos captados del público que se efectúen mediante valores, marco que incluye la actividad desarrollada por los emisores de valores. De ese modo, la norma, de acuerdo con la regulación financiera internacional ${ }^{11}$, prevé como objetivos de la intervención estatal en el mercado de valores: (1) la protección de los derechos de los inversionistas; (2) la promoción del desarrollo y la eficiencia del mercado de valores; (3) la prevención y el manejo del riesgo sistémico, y (4) la preservación de la confianza del público en el mercado. Tales objetivos propenden por fortalecer la relación determinada por la doctrina ${ }^{12}$ entre mercados financieros fuertes y economías fuertes.

Fradique-Méndez López, Carlos. "Emisores de valores”. En Régimen del mercado de valores, tomo II, 2017, 143-144.

10 Armour, John, Dan Awrey, Paul Davies, Luca Enriques, Jeffrey N. Gordon, Colin Mayer, Jennifer Payne, Principles of financial regulation, 163.

11 losco, Objectives and principles, 5.

12 Armour, John, Dan Awrey, Paul Davies, Luca Enriques, Jeffrey N. Gordon, Colin Mayer, Jennifer Payne, Principles of financial regulation, 54. 
Así mismo, en función de la eficiencia en el mercado de valores, la Ley 964 del 2005 establece como criterios de la intervención del Estado en este mercado, entre otros, que la regulación y la supervisión de este se ajusten a las innovaciones tecnológicas y faciliten el desarrollo de nuevos productos y servicios ${ }^{13}$, que los costos de la supervisión y la disciplina del mercado de valores sean eficientes y equitativamente asignados, que las cargas que se impongan a los participantes del mercado tengan en cuenta la comparación entre el beneficio que aportan y su costo $^{14}$, y que el mercado de valores esté provisto de información oportuna, completa y exacta ${ }^{15}$. Precisamente a estos objetivos y criterios se apelará con el fin de garantizar el cumplimiento del deber de información, de una forma interconectada y que evite duplicidades en la transmisión.

Como elemento fundamental de la regulación de los emisores de valores, los formuladores de política económica del mundo requieren de estas empresas un reporte de información obligatoria bajo la premisa usualmente implícita de que, en ausencia de dichas disposiciones, los precios del mercado no reflejarían la realidad económica de las empresas ni las utilidades previstas ${ }^{16}$. Sin embargo, el gran debate frente a la regulación de los emisores es que, en la búsqueda de mercados más eficientes, se requiere de una mejor revelación de información, lo cual implica un incremento en los costos generados por dicho cumplimiento y una consecuente disminución en la participación en el mercado ${ }^{17}$.

Lo anterior puesto que, aunque las empresas han demostrado ser conscientes de la necesidad de cumplir con las mejores prácticas de revelación de información, la implementación de este sistema de cumplimiento ha representado un esfuerzo adicional que no ha estado exento de críticas. Por otro lado, se ha argumentado que la innovación en la estructura de mercado es algo que debe promoverse, especialmente frente a regímenes de revelación de información en los que las cargas económicas resultan confiscatorias ${ }^{18}$. Así, han surgido normas que propenden por la innovación y por la estandarización como las Directivas sobre Mercados de Instrumentos Financieros de la Unión Europea (MIFID I y MIFID II) y los esfuerzos por

Congreso de la República de Colombia, Ley 964 del 2005, artículo 1, literal b, numeral 2.

Ibid., artículo 1, literal b, numeral 4.

Ibid., artículo 1, literal b, numeral 7.

Gelter, Martin. "Comparative corporate governance: Old and new”. 2017, 37-59., 53

Armour, John, Dan Awrey, Paul Davies, Luca Enriques, Jeffrey N. Gordon, Colin Mayer, Jennifer Payne, Principles of financial regulation, 165.

Wield, David Edward Kim y Lisa Newport. "Making stock markets work to support economic growth: Implications for governments, regulators, stock exchanges, corporate issuers and their investors", 15. 
establecer la adopción universal de los estándares de las normas internacionales de información financiera.

En ese orden de ideas, la regulación de los emisores ha representado una tensión entre la participación de las empresas en el mercado como emisores de valores, y los costos que derivan del cumplimiento de sus deberes y que tienden a desalentar esa participación ${ }^{19}$, al involucrar la contratación de personal encargado de recolectar y transmitir la información y las repercusiones económicas de tal revelación ${ }^{20}$. Lo anterior teniendo en cuenta que la revelación puede tener costos indirectos porque la información proporcionada al mercado de capitales puede ser utilizada por otras partes, por ejemplo, por competidores, sindicatos, reguladores, autoridades, etc. ${ }^{21}$.

De hecho, un estudio realizado ${ }^{22}$ demostró que algunos de los factores que desalientan a las empresas de listarse en bolsa son la revelación de su información a competidores. Este factor ha influido, junto con otros, en que las compañías tengan un enfoque prudente a la hora de tomar la decisión de listar sus valores en bolsa.

Dado que la actividad de los emisores implica la captación de recursos del público de manera significativa, y por lo tanto exige la confianza de los inversionis$\operatorname{tas}^{23}$, es oportuno que el deber de información pueda ser cumplido de una forma más eficiente. En ese contexto, las nuevas tecnologías ofrecen una solución a la tensión existente entre el cumplimiento del deber de información y la necesidad de mercados eficientes, permitiendo la entrada a nuevas empresas emisoras que cumplan sus deberes de información de una forma que aproveche la disponibilidad de las tecnologías de la información ${ }^{24}$.

\section{B) La regulación de los emisores de valores en Colombia}

De acuerdo con la regulación colombiana, los emisores de valores son las entidades que tienen valores inscritos en el Registro Nacional de Valores y Emisores (RNVE),

\footnotetext{
19 Hansen, Jesper Lau. "Say when: When must an issuer disclose inside information?",2016, 3.

20 Leuz, Christian y Peter D. Wysocki. "The economics of disclosure and financial reporting regulation: Evidence and suggestions for future research", 2016, 42.

21 Ibid., 36.

22 Brau, James C. y Stanley E. Fawcett. "Initial public offerings: An analysis of theory and practice", 2006, , 422-425.

23 Du Plessis, Jean Jacques, Anil Hargovan y Jason Harris. “Regulation of corporate governance”, $2018,15$.

24 Financial Stability Board (FSB) Financial Stability Implications from FinTech: Supervisory and Regulatory Issues that Merit Authorities' Attention, 2017, 22.
} 
sobre las cuales la Superintendencia Financiera de Colombia ejercerá control exclusivo, excepto en los casos en los que la entidad sea vigilada por otra autoridad, situación en la cual se tratará de un control concurrente ${ }^{25}$.

En virtud del control de la Superintendencia Financiera sobre los emisores, la supervisión de sus deberes corresponde a dicha superintendencia, bajo la aplicación de la Ley 964 del 2005, el Decreto 2555 del 201026, el Código de Comercio y la Circular Básica Jurídica de la Superintendencia Financiera. Con base en la regulación mencionada, los emisores de valores deben cumplir tres tipos de deberes que se encuentran íntimamente ligados entre sí: el deber de información, el deber de registro y los deberes de gobierno corporativo ${ }^{27}$.

El deber de información está íntimamente ligado a los deberes de registro y de gobierno corporativo. Por un lado, el deber de registro que implica la obligación de la empresa interesada en ser emisora, de inscribirse en el RNVE con el fin de ser autorizada para emitir valores en el mercado, supone que quien revela la información ha sido autorizado por la autoridad competente para emitir sus valores en el mercado, so pena de las sanciones previstas en la ley. Por otro lado, el deber de gobierno corporativo supone que los emisores demuestren a la autoridad financiera que cuentan con las herramientas necesarias para una adecuada administración y control de sus actividades ${ }^{28}$, las cuales hacen parte de la información que debe ser reportada por los emisores.

\section{C) Regulación del deber de información de los emisores de valores}

Para el caso colombiano, la supervisión del deber de información de los emisores de valores corresponde a la Superintendencia Financiera bajo la aplicación de la Circular Básica Jurídica y de la Circular Externa 004 del 2012 de esa superintendencia, además de las normas mencionadas para la regulación de los emisores.

Decreto 2555 del 2010, artículo 11.2.1.6.1.2.

Debe tenerse en cuenta que el Decreto 151 del 2021 recientemente modificó el contenido y la numeración de, entre otros, el Libro 2 de la Parte 5 del Decreto 2555 del 2010, relacionado con el régimen de revelación de los emisores. Para efectos del presente artículo, cuando se citen disposiciones que fueron modificadas por el mencionado decreto, se hará referencia, en un primer lugar a la numeración introducida por el Decreto 151 del 2021, y posteriormente, entre paréntesis, se citará la numeración del Decreto 2555 del 2010 anterior a la modificación. Esto, en atención a que en el régimen de transición introducido por el artículo 7 del Decreto 151 del 2021, los emisores de valores deberán cumplir las disposiciones dentro de los dos años siguientes a su publicación, esto es, el 10 de febrero del 2023. 
En específico, la Ley 964 del 2005 establece que el mercado de valores debe estar provisto de información oportuna, completa y exacta ${ }^{29}$. Con ese propósito, el Decreto 2555 del 2010 prevé como obligación de los emisores mantener permanentemente actualizado el RNVE remitiendo la información periódica y relevante a la Superintendencia Financiera y a los sistemas en los cuales se negocien dichos valores $^{30}$, de acuerdo con los plazos indicados en la Circular Externa 004 del 2012 de dicha superintendencia.

Por otro lado, el deber de información ha sido objeto de tratamiento por parte de la regulación internacional. En primer lugar, dado que la República de Colombia hace parte de la Organización para la Cooperación y el Desarrollo EconómiCOS (OCDE u OECD por su sigla en inglés), se tendrán en cuenta los principios para gobierno corporativo de dicha organización, que establecen de manera general que la revelación de la información contribuye a identificar y analizar la eficacia y la eficiencia de las decisiones de los órganos de dirección ${ }^{31}$. Así mismo, dado que la Superintendencia Financiera hace parte de la Organización Internacional de Comisiones de Valores (OICV o losco por su sigla en inglés), se han adoptado algunos de los principios de esta organización en lo que tiene que ver con la revelación completa, precisa y periódica de los resultados financieros, de los riesgos y de la información relevante ${ }^{32}$.

Información a revelar y reportar por los emisores de valores en Colombia

Como se mencionó, el deber de información se ha establecido como una herramienta para proteger a los inversionistas y para generar mercados eficientes en los que los precios de los valores se forman de acuerdo con la realidad del mercado y con la información disponible para los inversionistas. Así, la normatividad financiera colombiana, consciente de la necesidad de que los emisores doten a los grupos de interés de información actualizada y pertinente, estableció la obligación de revelar la información que se presenta a continuación.

En primer lugar, el emisor de valores debe divulgar, cuando tenga conocimiento de una situación relacionada con su emisión, la información relevante que habría sido tenida en cuenta por un experto prudente y diligente al comprar, Decreto 151 del 2021).

31 OECD, Corporate Governance Factbook, 2019, 14.

32 Iosco, Objectives and Principles, 22. 
vender o conservar sus valores o al momento de ejercer los derechos políticos inherentes a estos ${ }^{33}$. Frente a lo anterior, el Decreto 2555 del 2010, modificado por el Decreto 151 del 2021, contempla las siguientes categorías de situaciones que deben ser informadas recurrentemente por los emisores de valores: (1) su situación comercial y de sus operaciones ${ }^{34}$; (2) las situaciones de crisis empresarial del emisor; (3) sus entidades y/o patrimonios autónomos subordinados, o su entidad matriz ${ }^{35}$; (4) su situación financiera ${ }^{36}$; (5) los asuntos relacionados con la contabilidad y la información financiera del emisor ${ }^{37}$; (6) su Gobierno corporativo ${ }^{38}$; (7) su situación jurídica ${ }^{39}$; (8) información sobre la emisión y la negociación de valores ${ }^{40}$, y (9) cualquier evento que el emisor considere de importancia para sus inversionistas ${ }^{41}$.

Por otro lado, los emisores de valores deben remitir información periódica sobre los estados financieros de periodos intermedios y demás información que para el efecto establezca la Superintendencia Financiera, con la periodicidad y en los términos y condiciones ${ }^{42}$ previstos por esta en sus circulares externas 004 del 2012 (información relevante y de fin de ejercicio), 007 del 2015 y 011 del 2015 (información financiera de corte trimestral), y 018 del 2018 (composición accionaria). También, los emisores de valores deben presentar a la Superintendencia Financiera la información de fin de ejercicio que deberá incluir, al menos la documentación que debe someterse a consideración de la Asamblea General de Accionistas o del órgano que haga sus veces ${ }^{43}$.

Además, los emisores de valores inscritos en el RNVE tienen la obligación de remitir información periódica y relevante a la Bolsa de Valores de Colombia S. A. en los plazos y en los términos establecidos por la Superintendencia Financiera, y sin perjuicio de la obligación de ampliar o aclarar la información a solicitud de la bolsa.

Decreto 2555 del 2010, artículo 5.2.4.3.1 (antes artículo 5.2.4.1.5).

lbid., numeral 1 (antes artículo 5.2.4.1.5, literal c).

Ibid., numeral 2 (antes artículo 5.2.4.1.5, literal d).

Ibid., numeral 3 (antes artículo 5.2.4.1.5, literal a).

lbid., numeral 4 (antes artículo 5.2.4.1.5, literal a).

Ibid., numeral 5 (antes artículo 5.2.4.1.5, literal b. Este punto no se encontraba desarrollado en el Decreto 2555 del 2010 antes de la modificación introducida por el Decreto 151 del 2021).

Ibid., numeral 6 (antes artículo 5.2.4.1.5, literal b).

lbid., numeral 7 (antes artículo 5.2.4.1.5, literal e).

Ibid., numeral 8 (Este punto no se encontraba desarrollado en el Decreto 2555 del 2010 antes de la modificación introducida por el Decreto 151 del 2021).

Decreto 2555 del 2010, artículo 5.2.4.1.2 (antes artículo 5.2.4.1.3).

Decreto 2555 del 2010, artículo 5.2.4.2.2 (antes artículo 5.2.4.1.2). 
Así mismo, en el marco de esta revelación, los emisores deben contar con una página web en la cual se incluya información financiera, el prospecto de información de la emisión, los estados financieros, el Código de Buen Gobierno, las sanciones impuestas al emisor, entre otros ${ }^{44}$.

Por último, debe mencionarse que los emisores de valores, dado su deber de gobierno corporativo, deben remitir anualmente a la SFC el Reporte de Implementación de Mejores Prácticas Corporativas que tiene como objetivo permitir un conocimiento apropiado por parte de los grupos de interés sobre la marcha y la situación del emisor, así como aportar elementos de juicio suficientes para la toma de decisiones. Dicho reporte se encuentra fundamentado en el Código País y contiene medidas que se refieren a los derechos y trato equitativo de los accionistas, a la Asamblea General de Accionistas, a la Junta Directiva, a la Arquitectura de Control, y a la Transparencia e Información Financiera y no Financiera. Además, su reporte se basa en el principio de "cumpla o explique", lo que implica que en caso de que no se registre el cumplimiento de uno de los deberes, el emisor debe explicar el motivo de dicha ausencia ${ }^{45}$.

Dado este marco normativo, queda en evidencia que algunos elementos presentan similitudes entre sí. En primer lugar, situaciones contables que hacen parte de la información relevante, como el aumento o disminución del capital, la variación en el número de acciones en circulación, la aprobación de la distribución de dividendos por parte de la Asamblea de Accionistas o cambios en la clasificación contable de las inversiones, son elementos que pueden ser transmitidos mediante los estados financieros periódicos o de fin de ejercicio.

En el mismo sentido, el reporte de implementación de Código País incluye elementos de información relevante frente a la situación jurídica y a la situación comercial del emisor, lo cual permitiría que una vez transmitidas y reveladas las decisiones de la Asamblea de Accionistas o la imposición de sanciones al emisor o a sus administradores, esta información podría ser agrupada a los criterios de evaluación del emisor introducidos por el Código País, mediante técnicas automatizadas de organización de datos.

Por último, las herramientas de transmisión de información contribuyen también a que la información remitida a distintas autoridades, en este caso a la Superintendencia Financiera y a la Bolsa, sea debidamente compartida a través de un sistema de interconexión segura implementado mediante estas tecnologías. 
Esta interconexión será posible por medio de sistemas compartidos de la coordinación entre las autoridades en la creación de interfaces de programación de aplicaciones $^{46}$ (application programming interfaces en su idioma original o API), que son un conjunto particular de reglas y especificaciones que los software pueden seguir para interactuar entre sí. El estudio sobre las API y sus funcionalidades se profundizará más adelante en el texto.

El propósito de reportar la información relevante sobre un emisor y su emisión no debería comportar que esta se encuentre duplicada en la normativa mencionada, puesto que esta situación representa complejidades tanto para el emisor que debe enviar la misma información por distintos medios, como para el supervisor que recibe esta información de forma duplicada. Más aún, estas duplicidades son contrarias a los criterios de intervención estatal definidos en la Ley 964 del 2005. Dado lo anterior, la autoridad financiera debe propender por sistemas de información que recopilen los datos de una forma organizada y automatizada, que garanticen que esta sea entregada en una sola ocasión mediante un único módulo de información, y que se irradie, dentro del sistema del supervisor, para los distintos propósitos de la supervisión.

\section{Reflexiones sobre el cumplimiento del deber de información}

El cumplimiento del deber de información debe ajustarse a los requisitos y a las características establecidas por las autoridades, so pena de la imposición de sanciones. Así, el régimen sancionatorio del deber de información establece como una infracción al régimen legal del mercado de valores la no divulgación de la información que pueda afectar la colocación de valores, su cotización en el mercado o la decisión de vender, comprar o mantener dichos valores. Como se mencionó, la ley colombiana otorga a dicha divulgación las calidades de veraz, oportuna, completa y suficiente, y, por lo tanto, la infracción se puede configurar en casos en los que se omiten detalles relevantes de la información, situación que podría presentarse por un error operativo.

Dado lo anterior, el cumplimiento de dicho deber representa costos altos para los emisores de valores, tanto en lo que se refiere a la recolección de la información por profesionales especializados como al riesgo al que estos están expuestos en caso de transmitir información incompleta o imprecisa según el criterio de la 
autoridad competente ${ }^{47}$. Sin embargo, las consecuencias negativas que implica el registro en bolsa se equilibran con sus ventajas, como la fuente de financiamiento que representa la emisión, lo cual conlleva la realización de un estudio costo-beneficio para asegurar que adquirir la calidad de emisor representa para la compañía una verdadera ventaja en términos económicos y comerciales ${ }^{48}$.

Como consecuencia de esto, la utilización de sistemas interconectados de información que permitan una transmisión mucho más ágil y eficiente se ha presentado como una solución útil y adecuada a los requerimientos de la autoridad ${ }^{49}$. Sin embargo, se debe precisar que el esfuerzo innovador no debe recaer únicamente en los emisores, puesto que las autoridades también se encuentran obligadas a realizar una supervisión ajustada a las realidades tecnológicas evitando que esta implique cargas injustas ${ }^{50}$.

En vista de lo anterior, herramientas como el compliance, es decir, el esfuerzo de una empresa para asegurar que su comportamiento y el de sus agentes se ajustan a los requerimientos regulatorios, a las prácticas industriales y a sus políticas y normas internas, han resultado de gran utilidad para las empresas, propendiendo por un reporte preciso y eficiente a las autoridades ${ }^{51}$. No obstante, las tecnologías que se indicarán a continuación presentan mayores ventajas para lograr dicho cumplimiento, gracias a la superación de los sistemas tradicionales de supervisión y al cambio que proponen en la forma en que se remite la información a las autoridades ${ }^{52}$.

\section{El regtech como un instrumento para el cumplimiento regulatorio}

En vista de la problemática detrás del cumplimiento del deber de información, esta sección tiene como propósito presentar algunas de las tecnologías sobre las que se basa el regtech, y la viabilidad de su aplicación para el cumplimiento del deber de

47 Hansen, Say when, 3.

$48 \quad$ Ibid.

49 Bofondi y Gobbi, The big promise of fintech, 2017, 117.

50 Colaert, Veerle A. Regtech as a response to regulatory expansion in the financial sector, $2018,31$.

51 Root Martínez, The compliance process, 2019, 212-213.

52 Mainelli, Michael y Alistair K. L. Milne. "The impact and potential of blockchain on the securities transaction", 2003, 37. 
información, así como brindar un análisis crítico de las ventajas y los riesgos que conlleva dicha aplicación.

\section{A) ¿Qué es el regtech?}

La implementación de herramientas tecnológicas y de la ciencia de datos en el cumplimiento de la regulación financiera se ha presentado como una posibilidad para hacer más eficiente dicho proceso. Así, los reguladores y supervisores de varios países del mundo se han dado a la tarea de introducir elementos de la innovación financiera a los marcos de supervisión y esquemas de regulación con el fin de realizar procesos de regulación macroprudencial, garantizando el monitoreo del sistema financiero en su conjunto ${ }^{53}$.

Esta situación ha dado nacimiento al regtech, del cual han dado su definición distintos académicos y autoridades de supervisión a lo largo del mundo. Una definición extendida, y que ha sido objeto de varias críticas ${ }^{54}$, es la ofrecida por la Financial Conduct Authority (FCA), autoridad financiera del Reino Unido, que trata al regtech como un subconjunto del fintech ${ }^{55}$ enfocado en las tecnologías que pueden facilitar el cumplimiento de requerimientos regulatorios de forma más eficiente y efectiva que los mecanismos tradicionales. Desde otra perspectiva, la autoridad financiera colombiana ha definido el regtech como el aprovechamiento de los desarrollos tecnológicos para optimizar los procesos internos del regulador y del supervisor y reducir las cargas operativas para el sector financiero ${ }^{56}$.

El regtech tiene distintas funcionalidades, pero para efectos de este artículo se analizará su funcionalidad denominada complianceTech ${ }^{57}$, es decir, la utilización de software específicos en la transmisión de información que es producida y recolectada por diferentes áreas de una empresa, así como de sus subsidiarias. No obstante, debe agregarse que, tal como se verá más delante, el regtech tiene también la potencialidad de proveer asistencia técnica para que las entidades de supervisión y vigilancia creen y definan ambientes de prueba de nueva regulación, con el

53 Arner, Barberis y Buckley. "FinTech, regtech and the reconceptualization of financial regulation", $2016,400$.

54 Ibid., 383.

55 Fintech se refiere a innovación habilitada por la tecnología en servicios financieros, independientemente de la naturaleza o el tamaño del proveedor de los servicios (European Commission, FinTech: A more competitive and innovative European financial sector, 2017, 4).

56 Superintendencia Financiera de Colombia (SFC), Hacia la innovación sostenible y responsable en el sector financiero, 2018. 
propósito de determinar sus aciertos, así como los ajustes requeridos para que esta cumpla con su propósito ${ }^{58}$.

Para entender el funcionamiento general de las tecnologías que se presentarán a continuación, es necesario hacer referencia a la ciencia de datos toda vez que su elemento fundamental es la información y la forma en que esta pasa por un proceso de producción y recolección para después ser transmitida. En ese sentido, la ciencia de datos es el arte y la ciencia de adquirir conocimiento a través de datos, ya sea que se trate de datos estructurados, es decir, aquellos que se ordenan en una estructura que discrimina las observaciones a estos (filas) y las características de esa observación (columna), o de datos no estructurados, es decir, aquellos que deben analizarse más a fondo para ser ordenados ${ }^{59}$.

Por otro lado, un proceso relevante de la mencionada ciencia es la limpieza de los datos, en el que, una vez reunidos, se discriminan los datos más importantes que, desde un punto de vista técnico, merecen ser enviados. Este proceso es sin duda el más retador en términos intelectuales puesto que se trata de una actividad de resolución de problemas, y requiere de un trabajo de codificación con el fin de obtener la información en una forma limpia y ordenada, que facilite su visualización y análisis exploratorio ${ }^{60}$.

Así, la ciencia de datos estudia el proceso a través del cual los datos, una vez estructurados, producen información, y esta, una vez analizada, producirá conocimiento, condiciones que pueden ser desarrolladas mediante aplicaciones de inteligencia artificial que son capaces de predecir comportamientos mediante el análisis de la información ingresada ${ }^{61}$.

\section{B) Tecnologías regtech para el cumplimiento regulatorio}

En esta sección se describirán las tecnologías regtech que pueden ser aplicadas al cumplimiento del deber de información. Debe tenerse en cuenta, en primer lugar, que el uso de tecnología en el cumplimiento regulatorio no es un fenómeno exclusivo de las tecnologías regtech, dada su constante evolución. Por lo anterior, la conveniencia de las tecnologías presentadas a continuación estará sujeta a un análisis costo-beneficio, que podrá realizarse utilizando estas tecnologías en un ambiente

$58 \quad$ Ibid.

59 Ozdemir, Principles of data science, 2016, 26-27.

60 Cady, The data science handbook, 2017, 60.

61 European Commission, European financial stability and integration review 2019, 2019, 79. 
de prueba con el fin de determinar los objetivos, características, requisitos mínimos, mecanismos de participación y las entidades involucradas en la construcción de la nueva regulación que las implemente.

En lo relacionado con el cumplimiento del deber de información, debe resaltarse que las tecnologías que se describirán a continuación tienen dos elementos fundamentales en común. En primer lugar, permiten el almacenamiento y procesamiento de información de una manera más eficiente que las herramientas de supervisión tradicionales ${ }^{62}$. En segundo lugar, la automatización del proceso de regulación y de supervisión mediante estas tecnologías está direccionado a la sistematización de los datos recibidos por la autoridad de supervisión que fortalecen los sistemas de alertas tempranas y propenden por una verificación del cumplimiento de una forma integral ${ }^{63}$.

Una primera tecnología regtech útil para la transmisión de información son las application programming interfaces (API) o interfaces de programación de aplicaciones, que son un conjunto particular de reglas y especificaciones que los software pueden seguir para comunicarse e interactuar entre sí. El carácter abierto de las API, gracias a su publicidad, fomenta los estándares de integración y el uso innovador de su funcionalidad, útiles en el cumplimiento del deber de información pues evitan las duplicidades en la transmisión de la información ${ }^{64}$.

De ese modo, las entidades supervisadas pueden desarrollar y compartir las API, y los reguladores pueden crear API para envíos de cumplimiento junto con los acuerdos de nivel de servicio (service level agreements [SLA]) que garantizan la estandarización en la transmisión de la información. En el marco del cumplimiento del deber de información, las API tienen la potencialidad, de acuerdo con las pruebas piloto realizadas en el Reino Unido, de contener la información estandarizada que requieren las autoridades para cumplir su función de supervisión ${ }^{65}$.

También, se ha demostrado que la utilización de API abiertas, es decir, cuyo acceso es libre, contribuye al aprovechamiento de grandes cantidades de información, reduciendo las barreras de entrada al acceso. Esto permite abrir campo a las pequeñas y medianas empresas de tecnología para desarrollar servicios de valor agregado con base en la información que puedan conocer por medio de las API,

Ibid.

64 IIF, Regtech in financial services, 15. 
reduciendo las inversiones iniciales necesarias para proponer nuevas aplicaciones basadas en procesamiento de flujos ${ }^{66}$.

Otra tecnología útil para el cumplimiento del deber de información son los smart contracts, que son programas de software que ejecutan ciertas tareas si se presentan ciertas condiciones ${ }^{67}$. Estos programas tienen, por ejemplo, la potencialidad de recopilar y programar acuerdos, como aquellos a los que se llega en las reuniones de los órganos sociales. Así mismo, pruebas piloto como las realizadas en el Reino Unido han demostrado la posibilidad de establecer nodos virtuales para representar a las empresas reguladas y a los reguladores, mediante la creación de versiones ejecutables de la regulación mediante estos contratos inteligentes ${ }^{68}$.

La ejecución de los smart contracts funciona con tecnología blockchain, un tipo de tecnologías de registro distribuido (distributed ledger technology [DLT]) ${ }^{69}$ que, de forma descentralizada y autónoma, organiza los datos que le son introducidos en una cadena de bloques, en la que cada bloque, o nodo, contiene datos verificados, validados y luego encadenados al siguiente bloque. Este tipo de tecnología cuenta con códigos hash, es decir, códigos en bloque, para verificar que la información no ha sido alterada; con claves criptográficas, construidas a partir de códigos, para limitar el acceso a la información, y con un mecanismo de consenso que garantiza que solo se permita la entrada de la información esperada ${ }^{70}$.

La funcionalidad de encriptación del blockchain, dada la necesidad actual de realizar pruebas de su viabilidad y de los costos que representa, puede ser realizada también mediante tecnologías informáticas de encriptación y seguridad, como el data storage cell level security, que permite que solo la información relevante esté disponible para las personas, en función de su autorización de acceso, y que etiqueta cada componente de datos por propiedad, objeto y tipo de acceso, facilitando su búsqueda. Así mismo, las tecnologías biométricas, que habilitan el escaneo de huellas digitales e iris, el reconocimiento de rostros, y el reconocimiento remoto de documentos de identidad, son una alternativa para la seguridad de la información ${ }^{71}$.

66 Remolina, "Big Data: concepto y fundamentos”, 2020, 85.

67 Akgiray, "The potential for blockchain technology in corporate governance", 10.

68 DRR. Digital Regulatory Reporting, 9.

69 Las DLT consisten en un consenso de datos digitales replicados, compartidos y sincronizados distribuidos geográficamente en múltiples sitios, países o instituciones. No hay administrador central o almacenamiento de datos centralizado. (UK Government, Office for Science, Distributed Ledger Technology: Beyond blockchain, 2016, 17-18).

70 Reyes, Packin y Edwards, Distributed governance, 2017, 11.

71 IIF, Regtech in Financial Services, 12-13. 
Existen también alternativas de almacenamiento y procesamiento de la información como el big data, es decir, conjuntos de información que exceden la capacidad de una base de datos tradicional que tiene como propósito la captura, almacenamiento, manejo y análisis de la información $n^{72}$. Frente a esta funcionalidad, se encuentran también las analíticas de almacenamiento en la nube, es decir, una arquitectura tecnológica integrada que transmite y fusiona diferentes tipos de datos, con la potencia de la computación en la nube, esto es, en un espacio de almacenamiento de alta capacidad, con capacidades analíticas predictivas avanzadas ${ }^{73}$.

Por otro lado, la robotic process automation (RPA, o automatización robótica de procesos) consiste en el aprovechamiento de los sistemas basados en reglas para automatizar flujos de procesos comerciales repetibles y basados en la lógica, como la verificación de los controles internos de cumplimiento. Estos procesos se desarrollan mediante la intervención de bots, es decir, de sistemas automatiza$\operatorname{dos}^{74}$, los cuales facilitan o inician el trabajo de las demás tecnologías. Además, la automatización de los procesos y del flujo de trabajo facilita la ejecución eficiente de las tareas rutinarias, permitiendo que la información sea ordenada en secuencias integradas o flujos de trabajo inteligentes ${ }^{75}$, lo cual será útil en caso de transmitir una gran cantidad de información.

Una tecnología base para otras tecnologías es la inteligencia artificial (Al por su sigla en inglés), que consiste en la aplicación de herramientas computacionales para realizar tareas que tradicionalmente requieren de la sofisticación humana ${ }^{76}$, y es útil en el desarrollo de modelos de impacto de mercado, e incluso de cumplimiento, que describan sus efectos utilizando instrucciones anteriores como punto de partida ${ }^{77}$.

Dentro de los diferentes tipos de inteligencia artificial se encuentra el machine learning (o aprendizaje de máquinas) que implica la capacidad de los sistemas computarizados de aprender a través de los procedimientos y de las instrucciones

Bagnoli, The big data relevant market as a tool for a case by case analysis at the digital economy: Could the EU decision at Facebook/WhatsApp merger have been different?, 2017, 7. 2019, 3; Phillips, Collins, “Automation - It does involve people”, 2019, 1.

75 Locke, Bird, Perspectives on the current and imagined role of artificial intelligence and technology in corporate governance practice and regulation, 2020, 8.

76 Financial Stability Board (FSB). Artificial intelligence and machine learning in financial services Market developments and financial stability implications, 2017, 4.

77 Barberis, Arner y Buckley, The regtech book: The financial technology handbook for investors, entrepreneurs and visionaries in regulation, 2019, 237. 
impartidas por las personas que los programan. El machine learning es útil en la interpretación y aplicación de la nueva normatividad, y en la creación de un sistema de alertas tempranas ${ }^{78}$. Además, como se verá más adelante, el machine learning tiene la potencialidad de determinar patrones de conducta, útiles en la formulación de nueva normatividad, herramienta que permitirá determinar el impacto de la regulación y así realizar ajustes antes de su expedición.

Por otro lado, otra rama de la inteligencia artificial que puede ser usada para el cumplimiento normativo es el procesamiento de lenguaje natural (NLP, o natural language processing). Este consiste en un campo de las ciencias de la computación, que se encarga de procesar textos escritos o dictados por seres humanos ${ }^{79}$ y que tiene la potencialidad de poblar un modelo de datos en un sistema de reporte regulatorio ${ }^{80}$.

En fases avanzadas de la implementación de la tecnología NLP se ha explorado el desarrollo de regulaciones legibles por máquinas, iniciativa conocida como TechSprints desarrollado por la FCA, que tenía por objeto limitar las posibilidades de interpretación y la ambigüedad de las normas, a través del NPL y de la programación de regulaciones legibles por computadores ${ }^{81}$.

Una vez presentadas las tecnologías regtech que tienen potencialidades frente al cumplimiento del deber de información, se propondrá su aplicación para dicho cumplimiento de acuerdo con la sistematización presentada en la figura 1. Por un lado, la información pasará por un proceso de encriptación y codificación mediante tecnologías informáticas de encriptación y seguridad. Por otro lado, los smart contracts tendrán la capacidad de recopilar la información contenida en las decisiones de los órganos sociales y en los distintos acuerdos de la compañía, y las API tendrán la potencialidad de responder de forma automatizada a los requerimientos de la autoridad.

Así mismo, un sistema programado con machine learning y que, en un futuro, sea capaz de interpretar NLP, establecerá los patrones de cumplimiento contenidos en la información transmitida. En el marco de este proceso, los sistemas de RPA serán los encargados de transmitir y recibir la información, y el almacenamiento de la información podrá estar a cargo de tecnologías de big data y de almacenamiento en la nube.

Micheler, Whaley, Regulatory technology, 6.

Nay, Natural language processing and machine learning for law and policy texts, 2018,, 1-2

DRR. Digital Regulatory Reporting, 11.

81

Cermeño, "El uso de las nuevas tecnologías para el cumplimiento normativo", 2020, 372. 
Emisor

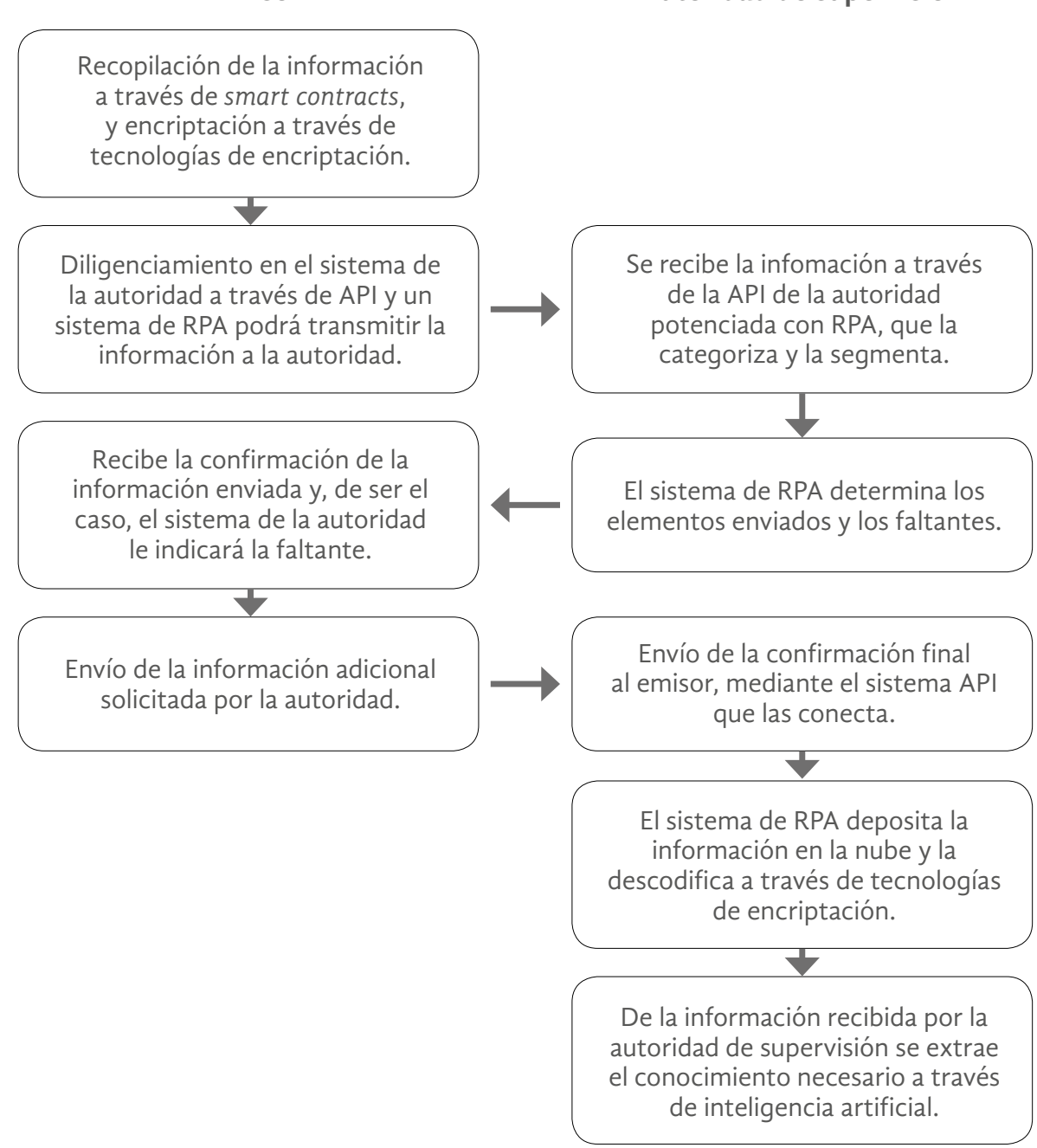

Figura 1. Mecanismo de implementación propuesto.

Fuente: el autor, con base en la información presentada a lo largo del presente artículo.

Frente a las tecnologías mencionadas, y el esquema de regulación propuesto en este artículo, debe mencionarse que los reguladores aún se encuentran en la etapa de participación abierta, donde están explorando los posibles beneficios y desafíos que ofrecen las soluciones regtech. A medida que los reguladores dan los primeros pasos hacia la adopción del regtech, se ha demostrado que encontrar pequeños casos de uso interno y probar nuevos enfoques en lugar de buscar únicamente soluciones de vanguardia, puede brindar mejores resultados.

Tal como se dijo, la propuesta presentada busca aprovechar los mejores elementos de cada una de las tecnologías para el cumplimiento del deber de información. Por lo tanto, la autoridad debe propiciar ambientes de prueba, los cuales, gracias a la aplicación de tecnologías basadas, por ejemplo, en la inteligencia artificial o en blockchain, permitan determinar la viabilidad de la nueva regulación. 
Lo anterior se propone toda vez que, para los reguladores, las tecnologías regtech permiten el desarrollo de herramientas de monitoreo continuo para identificar problemas a medida que desarrollan conocimiento, y reducen el tiempo que se tarda en investigar las violaciones del cumplimiento. En ese sentido, estas tecnologías fomentan el desarrollo de sistemas de simulación y de los sandbox, que pueden identificar las consecuencias probables de las reformas propuestas y los nuevos enfoques de regulación ${ }^{82}$.

Este ambiente de prueba, implementado en coordinación con la industria, demostrará la efectividad y la eficiencia que pueden brindar las tecnologías regtech a los procesos de cumplimiento regulatorio, así como las oportunidades de mejora y de corrección frente a los primeros pasos en aplicación de las tecnologías. Al centrarse primero en sus propios procesos, las agencias pueden obtener la experiencia práctica que necesitan con la nueva tecnología que está transformando el sector financiero, desde el big data y el aprendizaje automatizado hasta la computación en la nube y el blockchain, sin tener que preocuparse por exceder potencialmente sus poderes, o exponer a los consumidores o a los participantes del mercado al riesgo ${ }^{83}$, sin afectar materialmente procesos ni suspender requisitos.

Un área prometedora consistiría en probar las regulaciones "legibles por máquinas" que adjuntan etiquetas electrónicas a las reglas, con el fin de facilitar el seguimiento y la implementación de la regulación ${ }^{84}$. Es en este punto en que las tecnologías descritas pueden facilitar la predicción del impacto de la nueva regulación a expedir, con el propósito de que la autoridad que, en este caso, como se verá más adelante, será la SFC, pueda ajustarla antes de que sea efectivamente implementada. Los primeros pasos en este proceso pueden incluir automatización de procesos, pruebas basadas en conocimientos y modelos predictivos para priorizar el esfuerzo de supervisión ${ }^{85}$.

\section{C) Experiencia internacional con el regtech}

Las autoridades de supervisión del mundo se han interesado por la adopción de tecnologías para el cumplimiento normativo, dado que la confluencia de cambios regulatorios y tecnológicos relevantes a lo largo del tiempo ha creado incentivos

82 Arner, Barberis y Buckley, Fintech and Regtech, 20.

83 Barefoot, Banking needs a Regtech sandbox, 2018.

84 Ibid.

85 World Government Summit, Regtech for regulators: Re-architect the system for better regulation, 2018, 25. 
para las empresas y las entidades financieras sobre la forma en la que operan sus funciones de compliance ${ }^{86}$.

Algunas autoridades han entendido la necesidad de incluir este tipo de herramientas y procesos dentro de sus esquemas de supervisión y de regulación. En primer lugar, la Financial Conduct Authority ha promovido la adopción de tecnologías bajo dos frentes.

Por un lado, en la coordinación con otras autoridades de supervisión del mundo, a través de iniciativas como el Global Financial Innovation Network, de la que hacen parte importantes autoridades financieras del mundo, comprometidos con la integridad financiera, con la protección al consumidor financiero y con la estabilidad financiera, a través de la innovación ${ }^{87}$. Por otro lado, la FCA ha promovido la cooperación con la industria, a través de proyectos como el Digital Regulatory Reporting, que tiene como objetivo el compartir y sugerir ideas desde la industria y desde la supervisión ${ }^{88}$.

Así mismo, la FCA ha utilizado con éxito las tecnologías regtech en el marco de los ambientes de prueba, emitiendo informes reglamentarios en forma de código informático en lugar de palabras para permitir la auto implementación del cumplimiento, de una forma instantánea y económicamente viable.

Por su parte, la Autoridad Regulatoria de la Industria Financiera (Finra por su sigla en inglés) ha iniciado la discusión en Estados Unidos acerca del uso de las herramientas tecnológicas para los casos del compliance ${ }^{89}$. Esta autoridad ha tenido el propósito de mejorar las actividades de supervisión y otras funciones regulatorias y ha propendido por incluir en sus procedimientos mejoras en tecnología, tales como almacenamiento a través de la nube, analítica de big data y procesamiento de lenguaje natural.

También, algunas autoridades financieras como el Banco Central Europeo y la Reserva Federal de Estados Unidos están utilizando el procesamiento de lenguaje natural para identificar los riesgos a la estabilidad financiera, mediante la verificación de la correcta mitigación de los riesgos operacionales por parte de las entidades supervisadas ${ }^{90}$. El aumento en la utilidad del regtech se fundamenta principalmente en la crisis del 2008 que produjo un aumento en la regulación del

Finra, Technology based innovations, 2.

GFIN, GFIN - One year on, 3.

DRR. Digital Regulatory Reporting Pilot Phase 1 Report, 4.

El documento original se titula "Technology based innovations for regulatory compliance (Regtech) in the securities industry". https://www.finra.org/sites/default/files/2018_Regtech_Report.pdf 
gobierno corporativo, en el cual el compliance y la administración de los riesgos son, respectivamente, un elemento principal y una estrategia regulatoria crítica.

Así mismo, algunos gobiernos están tratando de digitalizar aspectos de la presentación de informes reglamentarios conectando a los reguladores e instituciones financieras directamente mediante API. Esto permitirá que ambas partes puedan monitorear las tendencias de riesgo utilizando datos completos, en tiempo real, al tiempo que reducen drásticamente los errores y gastos de presentación de informes ${ }^{91}$.

\section{D) Riesgos de la aplicación del regtech para el cumplimiento regulatorio}

A pesar de los beneficios que pueden aportar las tecnologías regtech, su aplicación no está exenta de críticas y de discusiones respecto de su idoneidad para el cumplimiento de los deberes regulatorios, entre ellos el de información. A continuación, se expondrán los tres riesgos más relevantes que han sido identificados frente a la aplicación del regtech para el cumplimiento de deberes normativos.

\section{Riesgo de ciberseguridad y seguridad de la información}

Las soluciones regtech garantizan que las entidades financieras y los emisores estén al día de los últimos retos regulatorios, minimizando la posibilidad de un error humano y asegurando el procedimiento corporativo en su totalidad, al tiempo que proveen una administración de riesgos novedosa y herramientas innovadoras sobre ciberseguridad. A pesar de estas ventajas, siempre que se presentan nuevas tecnologías para los diferentes procesos de cumplimiento regulatorio surge la preocupación de que la información cumpla con los estándares de seguridad de la información y ciberseguridad que eviten que la información transmitida sea utilizada de forma inescrupulosa por terceros.

Frente a los canales de transmisión de la información, la Superintendencia Financiera ha expedido normas que establecen que las entidades deben contar con políticas, procedimientos y recursos técnicos y humanos necesarios para gestionar efectivamente el riesgo de ciberseguridad ${ }^{92}$. En la doctrina internacional, algunas de las soluciones planteadas ${ }^{93}$ han sido entender que este no es un problema

91 Barefoot, Banking needs a Regtech sandbox.

92 SFC, Circular Externa 007 del 2018, numeral 3.

93 Dahlgren, The importance of addressing cybersecurity risks, 4-6. 
exclusivo de la tecnología, puesto que su solución debe basarse en la administración de riesgos, debe involucrar a la industria como un todo, y debe propender por una identificación correcta de las amenazas y las vulnerabilidades.

Así, se ha indicado que, si bien una mayor dependencia de la tecnología digital puede aumentar el riesgo de ciberseguridad, la tecnología digital también presenta numerosas oportunidades para mejorar la seguridad de los procesos de la empresa. Por ejemplo, el cifrado para proteger los datos almacenados digitalmente ha mejorado con la implementación de tecnologías de seguridad y encriptación. Así mismo, la implementación de tecnología biométrica permite mejorar la verificación y autenticación de la identidad, y el análisis de datos puede ser utilizado para detectar patrones irregulares y determinar si ha ocurrido fraude, además de permitir que las transacciones sean más fáciles de rastrear y de controlar ${ }^{94}$.

Por su parte, los controles automatizados emplean una variedad de mecanismos que limitan el campo de acción de los usuarios, reduciendo su discreción en la toma de decisiones. Los controles de acceso refuerzan las normas al prohibir la relación de diferentes tipos de información que deben permanecer independientes y asegurando que solamente los usuarios que están autorizados para ver determinada información puedan hacerlo efectivamente ${ }^{95}$.

\section{Riesgo frente a la sofisticación y a la calidad de la información}

Como sucede en los procesos de implementación de nuevas tecnologías, existe una latente preocupación de los reguladores y supervisores del mundo frente a la sofisticación y a la veracidad de la información transmitida, la cual surge principalmente porque no existe una verdadera garantía de su pertinencia, dado que no sería preparada por abogados. De hecho, el cumplimiento automatizado puede padecer de falta de rigor jurídico y de entendimiento de las consecuencias jurídicas de las distintas situaciones ${ }^{96}$.

Además, el cumplimiento del deber de información ha presentado complejidades en la transmisión de información recolectada y generada a través de sistemas informáticos. Esto dado que la programación con algoritmos, método mediante el cual funcionan los sistemas automatizados, puede presentar deficiencias en la transmisión de la información, como errores causados por la inaccesibilidad del

OECD, Financial markets, 14.

95 Bamberger, Technologies of compliance: Risk and regulation in a digital age, 2010, 692. 
código con el cual se generan las instrucciones, o dificultad en verificar la precisión y la veracidad de la información ${ }^{97}$.

Por otro lado, se argumenta que los procesos implementados dentro del cumplimiento sistematizado son privados y opacos. Dicha opacidad consiste en que el lenguaje utilizado por el programador no es revelado, y es extremadamente difícil comprender el tipo de datos que se recopilan. Así, esas capas de opacidad pueden ocasionar resultados de supervisión sesgados, discriminatorios o indeseables, como presentar como adecuada y verdadera información que fue transmitida parcialmente o de forma errónea, y hace que sea más difícil reconocer quién transmitió la información ${ }^{98}$.

Frente a lo anterior, la solución a esta problemática se ha encontrado en la coordinación entre los emisores y las autoridades y en la estandarización de los procesos, que garantice que la información será recibida por el sistema solamente si cuenta con los parámetros de transmisión definidos por la autoridad ${ }^{99}$. Este será uno de los elementos a tener en cuenta en la prueba de la regulación por medio de sandbox antes de su expedición.

En lo que se refiere a la sofisticación de la información jurídica y contable, transmitida mediante sistemas técnicos, la doctrina ha indicado que no debe exagerarse el entusiasmo de los científicos de datos ${ }^{100}$. Lo anterior puesto que, si bien los códigos son capaces de verificar y agregar decisiones individuales de larga escala sin intermediarios, es indispensable tener la capacidad de ver el mundo en su complejidad, así como aportar una fuerte dimensión ética y participación humana directa ${ }^{101}$.

Así las cosas, debe recordarse que ninguna tecnología es capaz de reemplazar totalmente equipos con criterio y conocimiento sofisticado del modo en la que se cumple el deber de información. En el mismo sentido, la tecnología tampoco tendrá la potencialidad de reemplazar una estructura de gobierno corporativo necesariamente sólida y que garantice la toma de decisiones en pro del interés y del desarrollo del emisor ${ }^{102}$. 


\section{Riesgo frente a los reguladores y supervisores}

Los reguladores deben explorar la posibilidad de utilizar tecnologías regtech para sus propósitos, con el fin de que el monitoreo y la supervisión del mercado de valores se realice mediante plataformas basadas en este tipo de tecnologías ${ }^{103}$, para lo cual deben contar con herramientas estandarizadas y con la más alta tecnología. Es así como, en un primer lugar, se ha argumentado que las autoridades deben tener acceso, por razones obvias, a las mejores tecnologías con el fin de interpretar y contrastar la información de una forma rápida y sin errores ${ }^{104}$.

En ese sentido, es evidente que las autoridades tienen la obligación de modificar sus estructuras con el fin de adecuarlas a generar un entendimiento autónomo y de captura de datos. Incluso, en el intento de combatir la opacidad de la tecnología escogida, estas estructuras administrativas garantizan una revisión desde varios puntos de vista, así como información sobre la situación que enfrentan los reguladores financieros que finalmente supondrá confiar en la intuición sobre la fuerza de la administración del riesgo privado ${ }^{105}$.

Por otro lado, es necesario que se adopten soluciones de estandarización para satisfacer los principios establecidos en cada regulación, lo cual es posible a través de la creación de API. Por este motivo se ha argumentado ${ }^{106}$ que los beneficios de una solución regtech unificada para todo el mercado representaría enormes eficiencias en la supervisión del cumplimiento de los deberes regulatorios y tendría la capacidad de superar los riesgos en el contexto del procesamiento de los datos por parte de la autoridad.

Además, la doctrina internacional ha exhortado a los formuladores de política pública a abandonar la regulación basada en reglas y enfocarse más en una supervisión basada en principios en conjunto con un monitoreo intensivo de datos contrastado con métricas cuantificadas ${ }^{107}$. En ese sentido, el regulador debe promover un cambio en el paradigma de cumplimiento, que trascienda la simple observancia de las reglas establecidas y que garantice la identificación de patrones de cumplimiento a través de herramientas como la inteligencia artificial ${ }^{108}$.

103 OECD, Blockchain technology and corporate governance, 2018, 22.

104 Atzori, Blockchain technology, 32.

105 Bamberger, Technologies of compliance, 734-735.

106 Colaert, Regtech as a response, 30.

107 King, Bank 4.0.: Everywhere never at a Bank, 2018, 86-87.

108 Arner, Barberis y Buckley FinTech, Regtech and the reconceptualization of financial regulation, 376. 
Un sector de la doctrina ${ }^{109}$, fundamentado en las predicciones de los científicos de datos, ha manifestado su preocupación frente al potencial reemplazo de los supervisores bancarios que la aplicación de estas tecnologías puede conllevar. Esta afirmación se podría sustentar dadas las ventajas obtenidas con los sistemas de supervisión basados en tecnologías como el machine learning, que utilizan técnicas matemáticas de optimización en comparación con los sistemas tradicionales de supervisión, basados en el conocimiento y las habilidades humanas. Sin embargo, un sector de la doctrina ${ }^{110}$ ha argumentado que tal situación además de estar lejos de la realidad es poco deseable puesto que la idea de Estado implica reconocer la necesidad de un punto de coordinación en la sociedad, el cual dirima de forma constructiva y política las tensiones entre el interés individual y el bien común.

Aunado a la necesidad de que el Estado sea el coordinador de la aplicación de estas tecnologías, debe tenerse presente que los reguladores, aprovechando la analítica de datos pueden obtener información valiosa mediante el estudio de patrones y la realización de intervenciones tempranas. Esto le permitirá utilizar las tecnologías mencionadas con el propósito de detectar oportunidades de mejora en la regulación a expedir, con la ventaja de corregir la regulación antes de que esta sea publicada y efectivamente implementada.

Dada esta potencialidad, el regulador tendrá la capacidad de entender la regulación como una plataforma ${ }^{111}$, lo cual permitirá que la regulación pueda ser evaluada antes de su expedición, utilizando tecnologías basadas en la inteligencia artificial y en el procesamiento de datos. Esto puede significar repensar el papel del regulador de múltiples formas, sin dejar nunca de lado su protagonismo en la formulación de normas que fortalezcan la supervisión del cumplimiento regulatorio.

\section{El regtech para el cumplimiento del deber de información en Colombia}

Dado el panorama regulatorio expuesto, esta sección presentará los retos relevantes de la aplicación del regtech en la legislación colombiana, así como las iniciativas recientes en este aspecto. Así mismo, expondrá las dificultades de dicha aplicación

109 Marden, The decline of politics, 2003, 90-91.

110 Atzori, Blockchain technology, 32.

111 World Government Summit, Regtech for regulators, 26. 
con base en lo indicado en la sección anterior, y propondrá una alternativa de aplicación ajustada a la realidad colombiana, por medio de una visión crítica del regtech.

\section{A) Aspectos relevantes de la aplicación del regtech en Colombia}

Los principales retos que la aplicación del regtech para el cumplimiento del deber de información en el ordenamiento jurídico colombiano, se resumen en los riesgos indicados en la sección anterior. Partiendo de la debida administración de tales riesgos, la autoridad de supervisión tendrá la capacidad de aprovechar el aumento de los datos disponibles, así como la capacidad potencial para acceder a y procesar estos datos, con el fin de realizar un monitoreo efectivo del cumplimiento regulatorio ${ }^{112}$.

De conformidad con lo anterior, gran parte de la doctrina ha considerado que la aplicación de tecnologías regtech implica un cambio de paradigma en el cumplimiento regulatorio, por cuanto conlleva retos sobre estructura de negocio y de entendimiento de los más altos estándares técnicos ${ }^{113}$. A pesar de estos retos, dicha aplicación hace posible la entrada de nuevos actores, comprometidos con el cumplimiento del deber de información y con una estructura sólida y eficiente atractiva para los inversionistas.

Las autoridades colombianas no han sido ajenas a la utilización de las herramientas de las tecnologías de la información y de la innovación financiera dentro de los procesos de supervisión de requerimientos regulatorios. En ese sentido, se han verificado diferentes iniciativas que tienen como propósito fortalecer la expedición de normas robustas y eficientes que permitan una supervisión de los requisitos de una forma ágil e interconectada.

En un sentido general, el Consejo Nacional de Política Económica y Social (Conpes) mediante el documento Conpes 3975 del 2019 -Política Nacional para la Transformación Digital e Inteligencia Artificial- introdujo un enfoque de gobernanza de la inteligencia artificial basado en riesgos que debe ser promovido dentro de las entidades del Gobierno y el sector privado ${ }^{114}$. Así, el mencionado documento está dirigido a impulsar la transformación digital de la administración pública y la promoción del desarrollo y gestión del talento para la transformación digital, iniciativas que deberán ser atendidas por las distintas autoridades, entre ellas la Superintendencia Financiera.

112 Iosco, losco Research Report on Financial Technologies, 2017, 71.

113 Packin, Regtech, compliance and technology judgment rule, 206-207.

114 Conpes, Documento Conpes 3975, 2019, 21. 
Por su parte, la Superintendencia Financiera, incluso antes de la expedición de dicho documento, ha realizado algunos acercamientos a la aplicación de las tecnologías regtech en sus procesos de supervisión y de regulación. A través de Innova SFC, el Grupo de Trabajo de Innovación Financiera y Tecnológica creado en el 2017, la Superintendencia Financiera ha impulsado, entre otros, la utilización de tecnologías regtech como una optimización del cumplimiento normativo, por medio de la cual se promueva la creación y desarrollo de aplicaciones que apoyen dicho cumplimiento ${ }^{115}$.

Adicionalmente, la Superintendencia Financiera mediante Circular Externa 029 del 2019, autorizó a las entidades vigiladas a adoptar tecnologías como las descritas para optimizar sus procesos, realizando una adecuada gestión de los riesgos asociados, verificando de manera regular la efectividad de los controles implementados y dando cumplimiento a las normas vigentes en materia de protección de datos y habeas data.

\section{B) Propuesta de aplicación de las tecnologías regtech en Colombia}

\section{Propuesta normativa}

Como se ha mencionado a lo largo de este artículo, la aplicación del regtech para los procesos de regulación, supervisión y cumplimiento normativo en Colombia requiere de un esfuerzo económico de los reguladores y de la industria, de un cambio en el paradigma de cumplimiento, y de una sofisticación y estandarización de los procesos. Ante su deber legal, las autoridades colombianas, en específico, la Superintendencia Financiera, deben considerar la utilización de tecnologías regtech que propendan por una intervención en el mercado de valores más eficaz y eficiente a través de una normatividad que asegure una utilización ordenada y con normas claras de transmisión.

Así, se propondrá un proyecto de normatividad, que debe ser guiado por la Superintendencia Financiera a través de una circular externa ${ }^{116}$ que establezca los canales precisos para la transmisión de la información, los cuales deben limitarse a los que actualmente existen, o incluso reducirse, de acuerdo con la tendencia

115 SFC, Nace innovasfc: espacio FinTech de la Superintendencia Financiera para apoyar, guiar y acompañar desarrollos tecnológicos para la industria financiera, 2018.

116 Las circulares externas son comunicaciones de carácter general, por medio de las cuales la Superintendencia Financiera imparte instrucciones y expide normas generales de imperativo cumplimiento para las entidades vigiladas, fija doctrina o manifiesta su posición institucional frente a temas de su competencia. 
mundial de integración y sistematización de la información transmitida. Esta circular debe tener como propósito derogar la Circular Externa 004 del 2012, que imparte instrucciones relacionadas con los reportes de información periódica y relevante, con el fin de homogenizarlos, tanto en lo que se refiere al reporte como a la recolección de la información. Estos procesos de sistematización se realizarán, de acuerdo con la presente propuesta, mediante las tecnologías descritas.

La circular propuesta, según la jerarquía normativa, es la norma idónea para la aplicación del regtech en Colombia, puesto que, como se ha mencionado, la Ley 964 del 2005 ya establece como criterio de la intervención estatal que las cargas que se impongan a los participantes del mercado tengan en cuenta la comparación entre el beneficio y el costo de estas. En segundo lugar, el Decreto 2555 del 2010 establece que le corresponde a la Superintendencia Financiera la determinación de los criterios y de los plazos en los que debe ser remitida la información. Frente a esto debe resaltarse que, si bien se propone un cambio en la forma en la que se concibe el deber de información, este se desarrollará en el proceso de recolección y transmisión, mas no en la determinación de la información a transmitir.

Como se ha mencionado, y en concordancia con el documento Conpes citado, la utilización de tecnologías de la ciencia de datos tiene la potencialidad de proveer asistencia técnica para que las entidades de supervisión y vigilancia creen y definan ambientes de prueba de nueva regulación a los que se ha hecho referencia, que permitan determinar los objetivos, características, requisitos mínimos, mecanismos de participación y las entidades involucradas en su construcción ${ }^{117}$. La utilización de estas tecnologías, que concretamente estará fundamentados en el machine learning y en los smart contracts asegurará que las normas, antes de ser expedidas, cumplan con los criterios técnicos y jurídicos para su adecuada ejecución ${ }^{118}$.

A continuación, se presenta un ejemplo de dicha transmisión, aplicado específicamente a la situación jurídica del emisor. Como se dijo, este aspecto comparte elementos que, con el sistema actual, deben ser transmitidos como información relevante, como información a la Bolsa de Valores, como información periódica, y como parte del reporte de implementación del Código País.

En primer lugar, el emisor debe verificar que la información que introduce, recopilada en su mayoría a través de smart contracts, cuente con las características y parámetros de encriptación y codificación establecidos por la autoridad, cuya 
actualización se realiza de forma automática en el sistema del emisor, mediante API que conectan las interfaces de lado y lado. Así, en caso de existir coincidencias en la información reportada y la información a reportar, el emisor, gracias a la estandarización de códigos garantizada por la coordinación con el sistema de la autoridad, no repetirá su transmisión, lo que ahorran tiempo y esfuerzos.

Tal sistema también permitirá la interconectividad con el sistema de la Bolsa de Valores, garantizando que, una vez cumplido el deber de información relevante ante la Superintendencia Financiera, se cumplirá también el deber frente a la Bolsa. Por otro lado, las API generadas por la autoridad permitirán determinar en tiempo real la relación existente entre la información aportada por el emisor ${ }^{119}$, y la información que requiere para realizar sus procesos de supervisión.

Dado lo anterior, los smart contracts permitirán la recopilación de la información de las decisiones de los órganos de dirección del emisor y las API, gracias a la interconexión con el sistema de la autoridad, de transmitir esa información estandarizada. Así, por ejemplo, datos que son recopilados con estas tecnologías, como las votaciones y las decisiones de la asamblea o la junta directiva del emisor ${ }^{120}$ y que son transmitidos por medio de las correspondientes actas podrán reposar en módulos de otro tipo de información, bien sea contable, financiera, comercial, entre otros.

Más concretamente, una vez se transmita la información sobre las decisiones de la Asamblea General de Accionistas, en la que se aprueba, entre otros, el proyecto de distribución de utilidades ${ }^{121}$, esta información además de incluirse como bloque de información a la cadena de la situación jurídica se unirá a la cadena de la situación contable y financiera, por ser la distribución de utilidades información relevante de este tipo. Además, las decisiones de la junta directiva ${ }^{122}$, que corresponden a información jurídica relevante, pueden versar sobre temas que son transmitidos dentro de esta, como los cambios en la composición accionaria iguales o superiores al cinco por ciento ( $5 \%$ ) de las acciones en circulación de la sociedad, actualmente entendidos como cambios significativos en la composición accionaria ${ }^{123}$, y que a su vez coinciden con información periódica de carácter financiero, como el reporte trimestral de la composición accionaria del emisor ${ }^{124}$.

119 Barberis, Arner y Buckley, The Regtech book, 131.

120 Lee, Distributed ledger technologies (blockchain) in capital markets: Risk and governance, 7.

121 Decreto 2555 del 2010, artículo 5.2.4.3.1, numeral 7.3.3 (antes artículo 5.2.4.1.5, literal a, numeral 5).

122 Decreto 2555 del 2010, artículo 5.2.4.3.1, numeral 5.4 (antes artículo 5.2.4.1.5, literal a, numeral 5)

123 Decreto 2555 del 2010, artículo 5.2.4.3.1, numeral 5.9 (antes artículo 5.2.4.1.5, literal b, numeral 1).

124 SFC, Circular Externa 018 del 2018. 
Por otro lado, la manera en la que es revelada la información jurídica relevante es información útil a la luz del Reporte de Implementación del Código País. Por ejemplo, una vez transmitida como información jurídica relevante la convocatoria a la asamblea ${ }^{125}$, estos datos se direccionarán al Reporte de Implementación de Código País, frente a la forma en la que está presentado el orden del día ${ }^{126}$ y a la forma en que pueden participar los accionistas en la reunión de la asamblea ${ }^{127}$.

Lo anterior se realizará sin necesidad de que el emisor deba enviar esta información cuando se encuentra diligenciando dicho reporte en los plazos establecidos para el efecto. Igualmente, información jurídica relevante como las decisiones de la asamblea de accionistas o de la junta directiva ${ }^{128}$ podrá direccionarse, en el caso de esta última, a medidas del Código País que se refieren a los mecanismos de reporte de información hacia la junta directiva y hacia la alta gerencia ${ }^{129}$.

Dadas esas relaciones, el sistema de la autoridad de supervisión, una vez recibida la información, enviará al emisor una notificación a través de RPA en la que se indiquen de forma precisa y fácilmente accesible para el funcionario encargado, los elementos que efectivamente fueron introducidos $y$, si los hubiere, los elementos pendientes por reportar.

Tan pronto se confirme al emisor que la información ha sido recibida en su totalidad, esta, mediante tecnología de inteligencia artificial, producirá el conocimiento requerido para desarrollar sus estrategias de supervisión, para contar con un panorama amplio de la situación de dichas empresas y para crear alertas tempranas respecto de las distintas situaciones del emisor. Así mismo, la información reposará en la nube de la autoridad, garantizando su disponibilidad y su seguridad.

En vista de lo anterior, la interconectividad permitirá que, si bien el emisor reportó información jurídica relevante, esta pueda ser incluida automáticamente a información periódica, a información contable y financiera y relevante, y al reporte de implementación del Código País. Así, queda en evidencia que la automatización permite que procesos que pueden tardar mucho y que con la normatividad actual se realizan en varias oportunidades, sean potencializados y cumplidos en un tiempo reducido Lo anterior garantizará un cumplimiento expedito y rápido por parte del emisor, y un monitoreo interconectado y en tiempo real por parte de la autoridad de supervisión.

Decreto 2555 del 2010, artículo 5.2.4.3.1, numeral 5.4 (antes artículo 5.2.4.1.5, literal b, numeral 1)

128 Decreto 2555 del 2010, artículo 5.2.4.3.1, numeral 2 (artículo 5.2.4.1.5, literal b, numeral 2).

129 SFC, Código País, medidas 28.2 y 28.3. 


\section{Administración de los riesgos del regtech}

La propuesta de regulación mencionada en la sección anterior debe ser estructurada de tal forma que responda adecuada y eficientemente a los riesgos que se determinaron en la sección "Riesgos de la aplicación del regtech para el cumplimiento regulatorio" de este artículo, frente a lo cual será de gran utilidad para el regulador la coordinación con la industria de sandbox en los que se pruebe de forma controlada la regulación antes de ser expedida. A continuación, se presentan las acciones y los mecanismos para que la autoridad de supervisión adecúe sus sistemas para cubrir los riesgos mencionados.

Las herramientas del regtech han demostrado que tienen potencial para desarrollar el sistema de cumplimiento regulatorio por parte de los emisores de valores, pues permiten una disminución de los costos de cumplimiento, y el fortalecimiento de la estructura de supervisión y regulación, pues dota a las autoridades de un campo de acción mucho más amplio. A pesar de estas eficiencias, el regtech requiere de un esfuerzo inicial, el cual debe ser analizado por los emisores y por los reguladores con el fin de conocer la verdadera eficiencia que aportan estas herramientas ${ }^{130}$.

En ese sentido, debe realizarse un análisis costo-beneficio que permita determinar los costos de las primeras etapas de la implementación del regtech en un país con limitaciones tecnológicas y de interconexión como Colombia, y compararlos con los beneficios que se derivarían de dicha aplicación. Por ese motivo, se recomienda que la implementación del regtech cuente con ambientes de prueba regulatorio que garantice una aplicación gradual, concertada y coordinada, tomando el ejemplo de otras jurisdicciones, con el fin de que tanto los emisores como las autoridades cuenten con el tiempo y los recursos suficientes para poder adaptarse al cambio.

Otro de los problemas que implica la utilización de tecnologías regtech para el cumplimiento del deber de información es la calidad y la sofisticación de la información que revelarán los emisores y que entregarán a las autoridades respectivas. La implementación de estas herramientas deberá ofrecer una verdadera interconectividad de la información, con el propósito de que esta sea más útil y pueda ser fácilmente clasificada por las autoridades para fortalecer los sistemas de supervisión y de alertas tempranas. 
Por este motivo, para eliminar la posibilidad de que los sistemas no cuenten con la información jurídica y financiera necesaria para recopilar y analizar los datos de manera adecuada, debe garantizarse, en primer lugar, que estos sistemas sean programados mediante una coordinación entre los equipos jurídicos y los equipos de tecnología de los emisores que aseguren un sistema de información que tenga en cuenta las necesidades del cumplimiento regulatorio y aporte la información requerida por el supervisor ${ }^{131}$.

En segundo lugar, con el fin de garantizar que los sistemas automatizados de información brinden y obtengan información útil y precisa, mitigando el riesgo de opacidad, se debe contar con una coordinación entre los emisores y las autoridades que garantice una estandarización de procesos que filtre la información, a través de API, y permita determinar si el emisor, con la transmisión realizada, cumplió con el deber de información. Dado lo anterior, tanto reguladores o emisores, en un esfuerzo por garantizar la veracidad de los datos transmitidos deben entender que más datos no necesariamente significan mejores datos ${ }^{132}$.

Por último, se propone la aplicación del regtech mediante tecnologías que velen por la seguridad de la información y que estén preparados para ataques cibernéticos de agentes maliciosos. Como se dijo, si bien la tecnología ha demostrado tener algunas falencias en temas de acceso y seguridad, esta tiene la capacidad de garantizar la seguridad por medio de soluciones como la encriptación, y el acceso al sistema central de la autoridad, limitado a los funcionarios encargados de analizar la información transmitida con procesos de identificación biométrica. Por ejemplo, herramientas fundamentadas en las tecnologías informáticas de encriptación y seguridad, garantizan que la información no será utilizada por terceros sin autorización ${ }^{133}$.

Si bien agentes maliciosos se han aprovechado del desarrollo tecnológico para capturar ilegalmente información valiosa, las autoridades, a través de la encriptación, han encontrado la solución de responder a ataques cibernéticos de gran magnitud ${ }^{134}$. Además, las autoridades han expedido normas que representan una respuesta regulatoria al auge de la digitalización de los servicios financieros, la mayor interconectividad de los agentes y la masificación en el uso de canales electrónicos, entre otros elementos, que han derivado en un incremento

131 Micheler, Whaley, regulatory technology, 12.

132 Remolina, Big Data, 92.

133 OECD, Financial markets, 18.

134 Packin, Regtech, compliance and technology judgment rule, 194. 
de la exposición a riesgos cibernéticos. Con ese propósito, la Circular Externa 007 del 2018 de la Superintendencia Financiera, exige, entre otros, la utilización de canales seguros para la realización de transacciones y para la transmisión de información, la implementación de actividades apropiadas para identificar la ocurrencia de un evento de ciberseguridad y la creación de procedimientos de respuesta a ataques cibernéticos.

\section{Conclusión}

Las innovaciones tecnológicas permiten que procesos que tradicionalmente son realizados por seres humanos puedan desarrollarse de formas más eficientes que permitan que la atención de estos se centre sobre temas sustanciales y de mayor valor económico, jurídico y comercial. Este artículo presentó la potencialidad de las tecnologías regtech para generar desarrollo en el mercado de valores y un aumento en el número de emisores, mediante una transmisión y revelación de información que, además de cumplir los requisitos, sea eficiente y se ajuste a las necesidades de los emisores. Así mismo, se recomendó la utilización de estas tecnologías para hacer pruebas de la regulación que las aplique antes de su expedición.

La aplicación de estas tecnologías, tanto en Colombia como en el mundo, requerirá de una transmisión de información ajustada tanto a las normas de la técnica y de la ciencia de datos como a las normas de la ética y de la transmisión de información veraz, útil y oportuna ${ }^{135}$, exigiendo una comunicación constante entre la industria y las autoridades. Es por ello que las tecnologías regtech no suponen que el Estado, en su función de supervisión de la actividad financiera, esté destinado a desaparecer, ni mucho menos que la transmisión de información no involucre conocimientos diferentes a los técnicos y científicos, como es el conocimiento jurídico.

Por este motivo, su aplicación, además de la necesaria coordinación, estará sujeta al involucramiento de funcionarios especializados en los temas a transmi$\operatorname{tir}^{136}$ y a un eficiente proceso de prueba y error como el que supone la utilización de cualquier innovación tecnológica. Esto con el fin de que la información transmitida cumpla con su propósito y permita una toma de decisiones eficiente y adecuada por parte de la autoridad que supervisa dicho cumplimiento.

135 Atzori, Blockchain technology, 22.

136 Phillips y Collins, Automation, 3. 
Con base en lo anterior, el presente artículo indicó que la aplicación de las herramientas del regtech para el cumplimiento del deber de información y su supervisión requiere de la expedición de una normatividad en la que se establezca la interconectividad de los datos recolectados por el supervisor y una estructura que supere los riesgos operacionales inherentes a dicha aplicación. Tal como se argumentó, dicha modificación normativa requerirá de una combinación de las tecnologías regtech en las distintas etapas del proceso de transmisión que aproveche las mejores características de cada uno, dada la taxonomía de datos subyacente.

El aprovechamiento de estas tecnologías para determinar la viabilidad de su uso, así como para asegurar su correcto funcionamiento en los escenarios de cumplimiento y supervisión, será crucial para determinar si el sistema financiero colombiano se vería efectivamente beneficiado de un proceso de innovación que involucre las tecnologías de la información presentadas, o si por el contrario debería comenzarse con su aplicación segmentada. Con todo, aplicar estas tecnologías supondrá cubrir y mitigar los riesgos inherentes a su utilización, escenario en el cual será muy útil la prueba que se realice de estas en espacios controlados como los sandbox.

Las innovaciones tecnológicas han sido una constante en los procesos de cumplimiento regulatorio y en la supervisión de este. El uso de herramientas que actualmente hacen parte del día a día en estos procesos, antes fueron considerados innovadores y despertaron objeciones. En la actualidad, el regtech y las tecnologías que lo desarrollan son innovaciones que tienen la potencialidad de cambiar la manera en la que se realiza y se entiende el cumplimiento regulatorio.

En ese sentido, su aplicación tanto en el escenario de cumplimiento regulatorio, como en las actividades que desarrollan los emisores, supone un compromiso por parte de las autoridades y de los emisores con el propósito de determinar los beneficios que pueden traer y la forma en que se enfrentarán los retos que involucran sus tecnologías. Este compromiso será crucial para que los sistemas de cumplimiento regulatorio se adapten cada vez más a una realidad en la que las herramientas tecnológicas son imprescindibles en los procesos que desarrollan los emisores de valores y las entidades vigiladas en general.

\section{Referencias}

Akgiray, Vedat. "The potential for blockchain technology in corporate governance". En OECD Corporate Governance Working Papers, n. ${ }^{\circ} 21$, OECD Publishing, París (2019): 10. https://pdfs.semanticscholar.org/ a75e/719ea904b47146f949c221e5d3f19101f319.pdf 
Armour, John Dan Awrey, Paul Davies, Luca Enriques, Jeffrey N. Gordon Colin Mayer y Jennifer Payne. Principles of financial regulation, 1st ed. Oxford: Oxford University Press, 2016.

Arner, Douglas W., Jànos Barberis y Ross P. Buckley. Fintech and regtech in a nutshell, and the future in a sandbox, CFA Institute Research Foundation, 2017.

Arner, Douglas W., Jànos Barberis y Ross P. Buckley. "FinTech, regtech and the reconceptualization of financial regulation". Northwestern Journal of International Law and Business, vol. 37, n. ${ }^{\circ} 3$ (2016): 376, 382, 383, 400. https://scholarlycommons.law.northwestern.edu/cgi/viewcontent. cgi?article $=1817 \&$ context $=$ njilb

Atzori, Marcella. Blockchain technology and decentralized governance: Is the state still necessary?, 2015, 22-32. https://papers.ssrn.com/sol3/ papers.cfm?abstract_id $=2709713$

Bagnoli, Vicente. The big data relevant market as a tool for a case by case analysis at the digital economy: Could the EU decision at Facebook/ WhatsApp merger have been different?, Ascola Conference 2017, 7. https://ssrn.com/abstract $=3064795$

Bamberger, Kenneth A. "Technologies of compliance: Risk and regulation in a digital age". Texas Law Review, vol. 88, 669 (2010); UC Berkeley Public Law Research Paper n. 1463727 (2010): 692, 733-735. https:// ssrn.com/abstract $=1463727$

Barberis, Jànos, Douglas W. Arner y Ross P. Buckley. The regtech book: The financial technology handbook for investors, entrepreneurs and visionaries in regulation. West Sussex: Wiley, 2019.

Barefoot, Jo Ann. "Banking needs a regtech sandbox". American Banker (blog). 13 de noviembre del 2018, https://www.americanbanker.com/ opinion/banking-needs-a-Regtech-sandbox

Bofondi, Marcello y Giorgio Gobbi. "The big promise of Fintech". European Economy, Issue 2017.2, (2017): 117. http://european-economy. eu/wp-content/uploads/2018/01/EE_2.2017-2.pdf

Bolsa de Valores de Colombia, Reglamento general.

Brau, James C. y Stanley E. Fawcett. "Initial public offerings: An analysis of theory and practice". The Journal of Finance, n. ${ }^{\circ} 61$ (2006): 422-25. doi:10.1111/j.1540-6261.2006.00840.x

Broeders, Dirk y Jermy Prenio. "Innovative technology in financial supervision (suptech) - the experience of early users". FSI Insights on Policy Implementation 9, 2018, 3. 
Cady, Field. The data science handbook. Hoboken, NJ: John Wiley y Sons, 2017.

Cermeño, Emmanuel. "El uso de las nuevas tecnologías para el cumplimiento normativo". En Aurelio Gurrea Martínez, Nydia Remolina, Fintech, regtech y legaltech: fundamentos y desafíos regulatorios. Madrid: Tirant Lo Blanch, 2020.

Colaert, Veerle A. Regtech as a Response to Regulatory Expansion in the Financial Sector (June 2018): 30-31. https://papers.ssrn.com/sol3/pa pers.cfm?abstract_id $=2677116$

Congreso de la República de Colombia. Ley 964 del 2005, Diario Oficial 45.963 del 8 de julio del 2005.

Consejo Nacional de Política Económica y Social (Conpes). Documento Conpes 3975: Política nacional para la transformación digital e inteligencia artificial, 2019, 21. https://www.mintic.gov.co/portal/604/arti cles-107147_recurso_1.pdf

Dahlgren, Sarah. The importance of addressing cybersecurity risks in the financial sector. Federal Reserve Bank of New York (March 24, 2015): 4-6. https://www.newyorkfed.org/newsevents/\%20speeches/2015/dah 150324

Deloitte. RegChain Reaction - Revolutionary reg. reporting in the fund's world, Issue n. ${ }^{0}$ 23, (2017): 3-4. https://www2.deloitte.com/lu/en/ pages/technology/articles/revolutionary-reg-reporting-in-the-fundsworld.html

Dhillon, Vikram, David Metcalf y Max Hooper. Blockchain enabled applications: Understand the blockchain ecosystem and how to make it work for you. Orlando: Apress, 2017.

Digital Regulatory Reporting (DRR). Digital Regulatory Reporting Pilot Phase 1 Report, 2018. https://www.fca.org.uk/publication/discussion/ digital-regulatory-reporting-pilot-phase-1-report.pdf

Enriques, Luca. "Financial supervisors and regtech: Four roles and four challenges". Revue Trimestrielle de Droit Financier, 53 (2017): 3, 5. https://papers.ssrn.com/sol3/papers.cfm?abstract_id=3087292

European Commission. "European financial stability and integration review 2019”, 2019, 79. https://ec.europa.eu/info/sites/info/files/euro pean-financial-stability-and-integration-review-2019_en.pdf

-. FinTech: A more competitive and innovative European financial sector. Consultation Document, 2017, 4. https://ec.europa.eu/info/sites/ info/files/2017-fintech-consultation-document_en_0.pdf 
Financial Stability Board (FSB). Artificial intelligence and machine learning in financial services Market developments and financial stability implications, 2017, 4. https://www.fsb.org/wp-content/uploads/P011117.pdf

-. Financial Stability Implications from FinTech: Supervisory and Regulatory Issues that Merit Authorities' Attention, 2017, 22. https://www.fsb. org/wp-content/uploads/R270617.pdf

Fradique-Méndez López, Carlos. "Emisores de valores". En Régimen del mercado de valores, tomo II. Bogotá: Temis y Ediciones Uniandes, 2017.

Gami, Manishkumar, Parth Jetly, Nidhi Mehta y Sunita Patil. "Robotic process automation-Future of business organizations: A review". 2nd International Conference on Advances in Science \& Technology (ICAST) 2019 on 8th, 9th April 2019 by KJ Somaiya Institute of Engineering \& Information Technology, Mumbai, India, 2019, 3. https://papers.ssrn.com/sol3/ papers.cfm?abstract_id $=3370211$

Gasparri, Giorgio. "Risks and opportunities of regtech and SupTech developments". Frontiers. Front. Artif. Intell. 2, 14 (2019): 2. doi: 10.3389/ frai.2019.00014

Gelter, Martin. "Comparative corporate governance: Old and new". En Understanding the company: Corporate governance and theory, editado by Barnali Choudhury y Martin Petrin, 37-59. Cambridge: Cambridge University Press, 2017.

Geslevich Packin, Nizan. "Regtech, compliance and technology judgment rule". Chicago-Kent Law Review, Forthcoming; Baruch College Zicklin School of Business Research Paper n. ${ }^{0}$ 2018-06-03 (2017): 194, 206-7, 215. https://papers.ssrn.com/sol3/papers.cfm?abstract_id=3043021

Global Financial Innovation Network (GFIN). GFIN- One year on. Reflects on its first year, 2019, 3. https://static1.squarespace.com/static/5db7cdf 53d173c0e010e8f68/t/5dbfaaca6b4e151deddc42ae/1572842207667/ GFIN-One-year-on-FINAL-20190612+\%28CLEAN+VERSION\%29.pdf

Hansen, Jesper Lau. "Say when: When must an issuer disclose inside information?". Nordic \& European Company Law Working Paper n. 16-03; University of Copenhagen Faculty of Law Research Paper n. ${ }^{\circ}$ 2016-28, (2016): 3. https://ssrn.com/abstract=2795993

Herrera, Diego y Sonia Vadillo. "Regulatory sandboxes in Latin America and the Caribbean for the FinTech ecosystem and the financial system". Inter-American Development Bank, Institutions for Development Sector Connectivity, Markets, and Finance Division Discussion Paper n. IDB-DP-573 (2018): 21. https://publications.iadb.org/publications/ english/document/Regulatory-Sandboxes-in-Latin-America-and-theCaribbean-for-the-FinTech-Ecosystem-and-the-Financial-System.pdf 
International Institute of Finance (IIF). Regtech in financial services: Solutions for compliance and reporting, 2016: 11-2, 23. https://www.iif.com/ Portals/0/Files/private/iif-Regtech_in_financial_services_-_solutions_ for_compliance_and_reporting.pdf?ver=2019-01-04-142943-690

International Organization of Securities Commissions (Iosco). Iosco Research Report on Financial Technologies. International Organization of Securities Commissions, 2017, 71. https://www.iosco.org/library/pubdocs/pdf/IOSCOPD554.pdf

Objectives and Principles of Securities Regulation. International Organization of Securities Commissions, 2010.

King, Brett. Bank 4.0: Everywhere never at a Bank. Singapur: Marshall Cavendish, 2018.

Lee, Joseph. Distributed ledger technologies (blockchain) in capital markets: Risk and governance, may 18, 2018, 7. https://papers.ssrn.com/ sol3/papers.cfm?abstract_id=3180553

Leuz, Christian y Peter D. Wysocki. "The economics of disclosure and financial reporting regulation: Evidence and suggestions for future research". European Corporate Governance Institute (ECGI) - Law Working Paper n. 306/2016 Chicago Booth Research Paper No. 16-03 (2016): 42. https://papers.ssrn.com/sol3/papers.cfm?abstract_id=2733831

Locke, Natania y Helen Louise Bird. "Perspectives on the current and imagined role of artificial intelligence and technology in corporate governance practice and regulation". Australian Journal of Corporate Law (2020): 2, 8. https://ssrn.com/abstract $=3534898$

Marden, Peter. The decline of politics. Ashgate: Aldershot, Hants, England, 2003.

Mainelli, Michael y Alistair K. L. Milne. "The impact and potential of blockchain on the securities transaction lifecycle". SWIFT Institute Working Paper n. ${ }^{\circ}$ 2015-007 (2016): 37. https://ssrn.com/abstract=2777404

Micheler, Eva y Anna Whaley. "Regulatory technology: Replacing law with computer code". LSE Law, Society and Economy Working Papers 14/2018, London School of Economics and Political Science, Law Department (2018): 6, 11-2, 16-7. http://eprints.Ise.ac.uk/89550/1/Micheler\%20SSRN-id3210962.pdf

Nay, John. Natural language processing and machine learning for law and policy texts. New York University School of Law, 2018, 1-2. https://papers.ssrn.com/sol3/papers.cfm?abstract_id=3438276 
Organization for Economic Co-operation and Development (OECD). Blockchain technology and corporate governance, directorate for financial and enterprise affairs Corporate Governance Committee, 2018, 22. https://www.oecd.org/officialdocuments/publicdisplaydocumentpd$\mathrm{f} /$ ?cote=DAF/CA/CG/RD(2018)1/REV1\&docLanguage $=$ En

- Financial markets, insurance and private pensions: Digitalisation and finance, 2018, 14-18. https://www.oecd.org/finance/private-pensions/ Financial-markets-insurance-pensions-digitalisation-and-finance.pdf

-. Corporate Governance Factbook. Organization for Economic Co-operation and Development, Directorate for Financial and Enterprise Affairs Corporate Governance Committee, 2019, 14. https://www.oecd.org/ corporate/Corporate-Governance-Factbook.pdf

Ozdemir, Sinan. Principles of data science. Birmingham: Packt Publishing, 2016.

Phillips, Delphine y Eddie Collins. "Automation - It does involve people". Business Information Review 36, n. 3 (2019): 1, 3. doi: https://doi. org/10.1177/0266382119863870

Plessis, Jean Jacques du, Anil Hargovan y Jason Harris. "Regulation of corporate governance". En Principles of contemporary corporate governance, 4th ed., 133-147. Cambridge: Cambridge University Press, 2018.

Remolina, Nydia. "Big Data: concepto y fundamentos". En Aurelio Gurrea Martínez, y Nydia Remolina, Fintech, regtech y legaltech: Fundamentos y desafíos regulatorios. Madrid: Tirant Lo Blanch, 2020.

Reyes, Carla L., Nizan Geslevich Packin y Benjamin P. Edwards . "Distributed Governance". William \& Mary Law Review Online, 59, n. ${ }^{\circ}$ (2017): 11. https://scholarship.law.wm.edu/cgi/viewcontent.cgi?article $=1003 \&-$ context $=$ wmlronline

Root Martínez, Veronica. "The compliance process". Indiana Law Journal, Notre Dame Legal Studies Paper n. ${ }^{\circ}$ 1838, 203 (2019): 212-213. https://papers.ssrn.com/sol3/papers.cfm?abstract_id=3151893

Superintendencia Financiera de Colombia (SFC). Circular Externa 018 del 2018.

—. Circular Externa 029 del 2019.

- Hacia la innovación sostenible y responsable en el sector financiero [Presentación], Santiago de Cali, 26 de abril del 2018. https:// www.superfinanciera.gov.co/descargas/institucional/pubFile1031001/ 20180426presinnovacali.pptx 
- Nace innovasfc: espacio FinTech de la Superintendencia Financiera para apoyar, guiar y acompañar desarrollos tecnológicos para la industria financiera), Santiago de Cali, 26 de abril del 2018. https://www.superfi nanciera.gov.co/jsp/10097387

—. Nuevo Código País. Circular Básica Jurídica (C. E. 029 del 2014), parte III, título I, capítulo V.

UK Government, Office for Science, Distributed Ledger Technology: beyond blockchain. (Report) (2016): 17-8. https://assets.publishing.ser vice.gov.uk/government/uploads/system/uploads/attachment_data/ file/492972/gs-16-1-distributed-ledger-technology.pdf

Wield, David Edward Kim y Lisa Newport. "Making stock markets work to support economic growth: Implications for governments, regulators, stock exchanges, corporate issuers and their investors". OECD Corporate Governance Working Papers, n. ${ }^{\circ}$ 10, OECD Publishing, París (2013): 15. https://www.oecd-ilibrary.org/docserver/5k43m4p6ccs3-en.pdf?expi res $=1589251633 \& i d=i d \&$ accname $=$ guest $\&$ checksum $=31$ D 550064EA 91207FEFB00190FD04654

World Government Summit. Regtech for regulators: Re-architect the system for better regulation, 2018, 25-26. https://www.worldgovernmentsummit.org/docs/default-source/publication/2018/9146_wgs_ accenturereport_english_fa_low-res5acf8ac4e97c6578b2f8ff0000a 7ddb6.pdf?sfvrsn=5eb3ef0a_0 